\title{
Heuristic Approach for Automated Shelf Space Allocation
}

\author{
Dario Landa-Silva* \\ ASAP Research Group \\ School of Computer Science \\ University of Nottingham, UK
}

\author{
Fathima Marikar ${ }^{\dagger}$ \\ School of Computer Science \\ University of Nottingham, UK
}

\author{
Khoi Le \\ ASAP Research Group \\ School of Computer Science \\ University of Nottingham, UK
}

\begin{abstract}
Shelf space allocation is the problem of efficiently arranging retail products on shelves in order to maximise profit, improve stock control, improve customer satisfaction, etc. Most work reported in the literature on this problem has focused on the case of large retailers such as big supermarkets. The interest here is to tackle this problem in the context of small retail shops where different issues arise when compared to large retailers. This paper proposes a heuristic approach to automate shelf space allocation in small retail shops. Several initialisation heuristics and local search moves are incorporated into the proposed method which generates high quality practical arrangements represented graphically as simple planograms.
\end{abstract}

\section{Categories and Subject Descriptors}

I.2.8 [Artificial Intelligence]: Problem solving, control methods and search, Heuristic methods

\section{Keywords}

Allocation Heuristic, Shelf Space Allocation, Planograms

\section{INTRODUCTION}

In this paper, we are interested in the computer generation of planograms which are the graphic representations of the arrangement of products on shelves in retail shops. We propose a heuristic approach for the automated allocation of shelf space. This approach has been developed in cooperation with a retailer who provided in-depth knowledge of the problem and requirements for a computer solution. We incorporate the heuristic approach into a prototype decision support system to aid managers of small retail shops

\footnotetext{
*Please send all correspondence to the following email address: dario.landasilva@nottingham.ac.uk

$\dagger_{\text {This }}$ work was carried out when Fathima Marikar was still at the University of Nottingham.
}

Permission to make digital or hard copies of all or part of this work for personal or classroom use is granted without fee provided that copies are not made or distributed for profit or commercial advantage and that copies bear this notice and the full citation on the first page. To copy otherwise, to republish, to post on servers or to redistribute to lists, requires prior specific permission and/or a fee.

SAC'09 March 8-12, 2009, Honolulu, Hawaii, U.S.A.

Copyright 2009 ACM 978-1-60558-166-8/09/03 ...\$5.00. to make better use of the limited shelf space. The heuristic approach provides an efficient arrangement of products into shelves and the graphical interface provides a graphical illustration of the proposed arrangement in the form of a simple planogram.

The goal of our research is to develop an algorithm to create an efficient arrangement of products on shelves or to improve upon an existing arrangement. The main objective considered here is to improve sales profit. For a realistic approach to understanding and solving the subject problem, we gathered real data and information from a small supermarket in the UN headquarters in Vienna, Austria. Previous work has focused on large retail shops such as big supermarkets (e.g. $[7,11,10,8])$. The heuristic method described in this paper consists of four phases: 1) the preparatory phase checks that enough shelf space in available for all products to be displayed, 2) the allocation phase constructs an initial arrangement, 3) the adjustment phase makes iterative changes to the arrangement in order to improve the overall profit, 4) the termination phase computes the quality of the final arrangement and generates the corresponding planogram.

Section 2 describes the shelf space allocation problem and gives an account of previous related research. Section 3 gives a formulation of the shelf space allocation problem in the small retail shop considered here. Section 4 describes in detail the four-phase heuristic algorithm proposed to tackle the subject problem. Section 5 presents and discusses our experiments and results while Section 6 concludes the paper.

\section{SHELF SPACE ALLOCATION}

Retailers require a competitive edge if they want to stay ahead of their competition. One way of doing this is to capture a larger market share and in the process, increase sales. One way of increasing sales is to improve retail operations and making an efficient use of the often limited shelf space in shops is an important strategy. Although shelf space allocation accounts for a small part on the whole set of management strategies for retailers, it is a comparatively easy way of earning more profits.

The management of shelf space in any retail outlet, big or small, plays a significant role in the shop's profitability [10, 8]. Shelf space is a vital yet limited resource and must be efficiently used and carefully managed in order to achieve the main objectives of maximising profits, reducing costs and increasing customer satisfaction. In their simplest form, the product selection problem refers to choosing which products are to be displayed in the limited shelf space available while 
the shelf space allocation problem refers to designing the actual arrangement of the chosen products on shelves and how many units of each product will be displayed [7].

There are many issues to consider when stocking shelves in retailing (see $[4,7,3,10]$ for more details). For example, items in clear view to the customer are the ones that are most likely to be seen and therefore bought. More importantly, a product must always be available on the shelf, otherwise loss of sales will occur. Products should be located depending on the target audience (e.g. toys and sweets should generally be placed on lower shelves so they are accessible to children). The larger the space given to a certain product, the easier it will be seen by customers. In many cases, there are standards imposed by the product manufacturers themselves (e.g. demanding that their product is located in certain shelves). Well-managed shelf space takes into consideration many of these issues and helps to achieve the objectives mentioned above in relation to profits, costs and customer satisfaction.

\subsection{Previous Work}

Several formulations have been proposed in the literature for the shelf space allocation problem. Previous research has focused on large supermarkets and therefore it is not fully applicable to small retail shops as the one considered in this paper. This is because not all data usually available for large retailers (e.g. sales, demand, marketing) is available for smaller ones. Examples of models for the shelf space allocation problem include those proposed in $[4,1$, $7,5,3,11,6,2,10,9,8]$. Cairns proposed an interactive approach to produce a graphical model taking into account factors such as space elasticity with the main objective of maximising profits [4]. Space elasticity is a common concept in shelf space management and it basically refers to the ratio of change in unit sales of a product, in relation to the change in shelf space allocated to the product (see [6]). Anderson and Amato investigated the role of consumer preference for specific product brands on the potential product demand and hence the impact on deciding the optimum allocation of shelf space [1]. Later, Hansen and Heinsbroek proposed an improved model which considered the cost effect [7]. In the model by Corstjens and Doyle the objective was to optimise profits considering three main issues: the cost effect, the demand effect and the cross elasticity effect [5]. Cross elasticity refers to the effect that sales of certain products can have on sales of other products. The model by Buttle incorporates five factors: fixture location, product category location, item location within categories, off-shelf display and promotional support [3]. Buttle suggests that all these aspects must be taken into consideration in order to increase profits. Zufryden followed in the footsteps of Corjstens and Doyle and produced a dynamic model including many demand-related marketing variables but not cross-elasticity among other simplifications [11]. Dreze et al. suggested that while location plays a significant role in the arrangement of products, the number of facings of each product was far less important (i.e. after a certain number, an additional facing does not make any difference to the product sales) [6]. A facing represents one unit of the product face straight out towards the customer. Borin et al. suggested that product assortment (selecting which products to display) and stockouts (products missing from shelves) are crucial issues that must be considered in shelf space manage- ment in addition to the demand effects [2]. The elaborate model by Yang and Chen incorporates many of the concepts from previous models and new ones, e.g. space elasticity, cross elasticity, marketing variables (price, promotion, advertisement, etc.), demand effect, display costs, inventory levels, etc. [10]. More recent studies tried to simplify the complex nature of previous models without compromising the ease of application. For example, Yang proposed a simple linear model based on the knapsack problem and only considering the profitability of products [9] and showed that the problem is NP-hard. Lim et al. proposed an extended model considering the effect of product groupings, i.e. placing products of the same category together or apart, and the effect of non-linear profit functions [8].

It is clear that the optimisation of shelf space allocation is very important and several models have been proposed. Most of the work carried out so far concentrates on large retailers and takes into consideration various marketing and consumer behaviour variables, which are not completely relevant or not available in the case of small retail shops. Small retailers are more likely to benefit from a model that is focused on their specific needs and takes into account constraints, requirements and rules that are specific to small shops. The simplified (yet NP-hard) models proposed more recently by Yang [9] and Lim et al. [8] seem more applicable to small retail shops. The formulation used in this paper is a modification of the one by Yang [9].

\section{PROBLEM SPECIFICATION}

The small shop considered in this study organises shelves into blocks, where each block consists of a set of shelves and each shelf can be divided into parts. A shelf is considered a horizontal unit of space, i.e. a shelf can be top-level, eyelevel or bottom-level. Each shelf is then split horizontally into left, middle and right parts of the shelf. Different priority is assigned to shelves and to parts within the shelf. Based on the examination of sales history, the eye-level shelves and centre shelf parts are assumed to have the highest profitability. For each product, the following is known: the minimum and maximum number of facings required, the length of one facing, the total number of units available, the profit per unit and the profit category code. The height of each product unit is ignored because the practice in the shop is to stack two units of products one above the other and the height of all shelves is enough to allow this for all products. The shop management does not require a product selection stage to decide which products should be displayed on shelves. The shop does not generally place facings of the same product on different shelves. However, they stated that if doing so improves profitability then they would be willing to try that kind of arrangement. The shop management groups products by general categories (for example, tea, coffee, cereals) but more sophisticated product grouping methods are not used during allocation. The reason for this is that the shop is small and products are found easily by customers because shelves are close enough to each other.

The problem formulation is as follows:

$$
\begin{aligned}
& n: \text { number of products to allocate } \\
& m: \text { number of shelves } \\
& p: \text { number of parts in each shelf (in our case } p=3 \text { ) } \\
& a_{i}: \text { length of facing of product } i
\end{aligned}
$$


$T_{j k}:$ length of part $k$ in shelf $j$

$L_{i}$ : minimum number of facings required for product $i$

$U_{i}$ : maximum number of facings required for product $i$

$x_{i j k}$ : number of facings of product $i$ on part $k$ of shelf $j$

$y_{i j k}=1$ if facings of product $i$ are assigned to part $k$ of shelf

$j, 0$ otherwise

$\rho_{i}$ : profit per unit of product $i$

$\sigma_{j}:$ priority coefficient of shelf $j$

$\omega_{k}:$ priority coefficient of shelf part $k$

$\varphi_{i j k}=\rho_{i} \sigma_{j} \omega_{k}:$ profitability of product $i$ if placed on part $k$ of shelf $j$

$i=1 \ldots n, j=1 \ldots m, k=1 \ldots p$

The objective is to maximise the total profit given by:

$$
Z=\sum_{i=1}^{n} \sum_{j=1}^{m} \sum_{k=1}^{p} \varphi_{i j k} x_{i j k}
$$

Subject to the following constraints:

$$
\begin{gathered}
\sum_{i=1}^{n} a_{i} x_{i j k} \leq T_{j k} \text { for } j=1 \ldots m, k=1 \ldots p \\
\sum_{j=1}^{m} \sum_{k=1}^{p} y_{i j k}=1 \text { for } i=1 \ldots n \\
L_{i} \leq \sum_{j=1}^{m} \sum_{k=1}^{p} x_{i j k} \leq U_{i} \text { for } i=1 \ldots n \\
y_{i j k} \leq x_{i j k} \leq U_{i} y_{i j k} \text { and } x_{i j k} \in \mathcal{Z}^{+}
\end{gathered}
$$

The following assumptions are made in the formulation:

1. There is enough shelf space available for the minimum number of products allocated, so that a selection algorithm is not required.

2. Product and shelf height and depth are ignored (only number of facings are considered).

3. Products are allocated adjacently (not stacked on top of each other).

4. All facings of a product must be placed on a single shelf (facings of a product cannot be placed on different shelves).

Note that the model above is an adaptation of Yang's model [9] to allow priorities for shelves and shelf parts to reflect current practice in the UN shop (and other small retail shops) where long shelves tend to be divided into smaller parts for better management. Allowing the shop manager to divide a shelf into parts and assigning a priority based on their observations about shelf profitability results in a more accurate overall profit estimation. A potential difficulty of dividing a shelf into parts arises when a product cannot fit into the remaining space in a shelf part. In this case the product might be assigned to another shelf part leaving unused space in the first shelf part. This could result on the fragmentation of space in the shelf parts which is less likely to occur if the whole shelf is treated as a unit.

\section{PROPOSED HEURISTIC APPROACH}

\subsection{Yang's Heuristic Method}

Yang proposed a three-phase heuristic to tackle the shelf space allocation problem in large retails shops [9]. We first outline Yang's method below and in the next subsection we explain our proposed adaptation.

1. Preparatory phase. Checks that solving the current problem is feasible, i.e. that there is enough shelf space to allocate the minimum required number of facings for all products. If this is the case, a set of priority indexes based on the notion of 'profit weight' is created. The 'profit weight' is calculated by dividing the profitability of product $i$ with respect to each shelf $k\left(P_{i k}\right)$ by the length of the product's facing $\left(a_{i}\right)$. Products with larger 'profit weight' are given a higher priority index. The algorithm continues to the next phase if solving the problem is feasible, otherwise it stops.

2. Allocation phase. Uses two sub-phases to construct an initial arrangement by assigning shelf space to each product following the ordered priority indexes established in the preparatory phase. This allocation phase tries to allocate a number of facings of each product within the given lower $\left(L_{i}\right)$ and upper $\left(U_{i}\right)$ bounds. As noted by Yang, assigning shelf space following the priority indexes does not guarantee that a feasible arrangement can be constructed, the whole algorithm might fail in this phase.

3. Adjustment phase. If a feasible initial solution is created in the allocation phase, changes are made to improve the arrangement by swapping the location of products and/or adjusting the number of facings. Yang proposed three adjustment methods. The first adjustment method swaps one facing between two products allocated to the same shelf. The second adjustment method swaps one facing between two products allocated to different shelves. The third adjustment method (extension of second method) seeks additional space in a shelf to allocate more facings of a product before swapping facings between two products in different shelves.

4. Termination phase. Calculates the total profit of the final arrangement.

\subsection{Adaptations Made in Our Approach}

We now give an overview of our method highlighting the differences with Yang's approach. After that, the next subsection gives full details of these different elements in our method.

1. Preparatory phase. Checks that solving the current problem is feasible but we do not create the list of priority indexes proposed by Yang.

2. Allocation phase. Creates an initial solution by one of three optional methods based on different criteria: product profit, product size and random choice. Alternatively, the user can provide an existing arrangement and apply phases 3 and 4 only. Yang used only one initialisation method based on product weight as described above. 
3. Adjustment phase. Consists of five heuristic moves designed to improve upon the current solution. These heuristics swap products between shelves based on different criteria. Only one of these heuristics is inspired by Yang's work.

4. Termination phase. As in Yang's method, this phase calculates the total profit for the final arrangement but we also implemented a graphical user interface for the shop manager to visualise the proposed arrangement in the form of a simple planogram.

\subsection{Initialisation in the Allocation Phase}

We implemented three optional initialisation methods in the allocation phase. The three methods place one product at a time on a given shelf part until all products are allocated. The methods differ in the order in which the products are sorted.

Arrangement Profit. This is a modified version of the initialisation algorithm proposed by Yang and it consists of three steps:

1. Prioritise. Products and shelves are sorted such that the most profitable products are assigned to the most profitable shelf parts. Products are sorted in descending order of their profit weight $\left(\rho_{i} / a_{i}\right)$ which represents the profit of a product relative to its size. All $(p \times m)$ shelf parts are sorted in descending order of their priority coefficients $\left(\sigma_{j}\right.$ and $\left.\omega_{k}\right)$.

2. Allocate Minimum. Using the sorted lists from step 1, select the first product and first shelf part and allocate the minimum required number of facings of the product $\left(L_{i}\right)$ to that shelf part. Repeat this process for the next product and next shelf part. After the last shelf part in the last shelf is reached, continue with the first shelf part in the priority list until all products have been allocated. This step ensures that $L_{i}$ facings of each product are placed in the shelves and remaining space is likely to exist in all shelf parts.

3. Allocate More. Using the arrangement from step 2 and the product priority list from step 1, increment the number of facings of each product by one ensuring that the upper bound on the number of facings $\left(U_{i}\right)$ and the length of the shelf part $\left(T_{j k}\right)$ are not exceeded. Continue incrementing the number of facings by one until no more facings can be allocated.

Note that the initial solution method based on profit implemented by Yang [9] requires the profit of a product $i$ with respect to each shelf $k$. However, the product profitability values $\left(\rho_{i}\right)$ obtained from the UN shop are independent of the shelf allocation. Also, the priority coefficients obtained for shelves $\left(\sigma_{j}\right)$ and shelf parts $\left(\omega_{k}\right)$ are independent of the product allocated.

Arrangement Size. This method is similar to the arrangement by profit but the criteria for ordering products is the facing length.

1. Prioritise. Products and shelves are sorted such that the most profitable shelf parts are assigned as many products as possible. Products are sorted in ascending order of their facing length $\left(a_{i}\right)$. All $(p \times m)$ shelf parts are sorted in descending order of their priority coefficients $\left(\sigma_{j}\right.$ and $\left.\omega_{k}\right)$.

2. Allocate Minimum. Idem Arrangement Profit step 2.

3. Allocate Rest. Idem Arrangement Profit step 3.

Arrangement Random Choice. This method serves as a benchmark to assess the usefulness of the product priority list used in the above two methods. All $(p \times m)$ shelf parts are sorted in descending order of their priority coefficients $\left(\sigma_{j}\right.$ and $\left.\omega_{k}\right)$ but products are not sorted.

1. Allocate Minimum. Randomly select a product and allocate the minimum required number of facings $\left(L_{i}\right)$ of the product to the first shelf part in the priority list. Continue with another randomly selected product and the next shelf part in the priority list. After the last shelf part in the last shelf is reached, continue with the first shelf part in the priority list until all products have been allocated.

2. Allocate Rest. Idem Arrangement Profit step 3.

User-defined Arrangement. We introduced this option to allow users to improve upon their existing arrangement. The arrangement is designed and supplied by the user in the form of a text file which should include a list of all products with the following details: shelf code, shelf part code, product code and currently allocated number of facings. In this study we considered the actual arrangement of products in the UN shop.

\subsection{Heuristic Moves in the Adjustment Phase}

The moves proposed by Yang [9] can result in facings of the same product being placed on different shelves which is not allowed in our case. We introduce the AdjustInter move to carry out swaps between four products located on two different shelves. This move makes possible to examine different neighbourhoods to possibly improve the overall solution. The RemoveLeastProfitable move is also introduced to escape from local optima. This move was initially designed to fully remove the least profitable product from every shelf part. However, after some preliminary testing, we observed this was too much and it would greatly reduce the overall profit if many shelf parts. We now describe the five heuristic moves used in the adjustment phase of our approach. Four of these heuristic moves aim to improve profit by changing the location or the number of facings of products in shelves. The fifth move disturbs the current arrangement by releasing shelf space and the aim is to diversify the search.

\section{Swap}

This move swaps all facings between two products $i_{1}$ and $i_{2}$ located on different shelf parts $j_{1} k_{1}$ and $j_{2} k_{2}$ respectively. The aim is to improve the overall profit by moving high profitable products to more profitable shelves. The following must be satisfied: $\Delta T_{j_{1} k_{1}}+a_{1_{1}} x_{i_{1} j_{1} k_{1}}-a_{i_{2}} x_{i_{2} j_{2} k_{2}}>0$ and $\Delta T_{j_{2} k_{2}}-a_{1_{1}} x_{i_{1} j_{1} k_{1}}+a_{i_{2}} x_{i_{2} j_{2} k_{2}}>0$.

\section{AdjustIn}

This move was proposed by Yang [9] and it adjusts by 1 the number of facings of two different products $i_{1}$ and $i_{2}$ on the same shelf part $j k$. The move makes $x_{i_{1} j k}=x_{i j k}+1$ 
and $x_{i_{2} j k}=x_{i_{2} j k}-1$. The aim is to improve the overall profit by adjusting facings so that high profitable products have more facings displayed. The following must be satisfied: $\Delta T_{j k}-a_{i_{1}}+a_{i_{2}}>0, x_{i_{1} j k}<U_{i_{1}}$ and $x_{i_{2} j k}>L_{i_{2}}$.

\section{MultiShift}

This move was proposed by Lim et al. [8] and is an extension of the AdjustIn move that allows an adjustment of multiple facings between two different products $i_{1}$ and $i_{1}$ ) on the same shelf part $j k$. The move adjust facings of both products by randomly choosing values $\alpha_{1}$ and $\alpha_{2}$ so that $x_{i_{1} j k}=x_{i_{1} j k}+\alpha_{1}$ and $x_{i_{2} j k}=x_{i_{2} j k}-\alpha_{2}$. Like AdjustIn, the aim of MultiShift is to improve the overall profit in the arrangement by adjusting facings so that high profitable products have more facings displayed. The following must be satisfied: $\Delta T_{j k}-\alpha_{1} a_{i_{1}}+\alpha_{2} a_{i_{2}}>0, x_{i_{1} j k}+\alpha_{1} \leq U_{i_{1}}$ and $x_{i_{2} j k}-\alpha_{2} \geq L_{i_{2}}$.

\section{AdjustInter}

We propose this move to make possible swaps between four different products located on two different shelf parts. The aim is to improve the overall profit by adjusting facings between the first pair of products in the hope that a profit improving move can be made later with the second pair of products. For products $i_{1}$ and $i_{2}$ on shelf $j_{1} k_{1}$ and products $i_{3}$ and $i_{4}$ on shelf $j_{2} k_{2}$, there are two possibilities:

1. Interchange $i_{1}$ (on shelf $j_{1} k_{1}$ ) and $i_{3}$ (on shelf $j_{2} k_{2}$ )

If $x_{i_{1} j_{1} k_{1}}=L_{i_{1}}$ and $x_{i_{3} j_{2} k_{2}}=L_{i_{3}}$, and there is enough space on each shelf part to swap $i_{1}$ and $i_{3}$, make the swap, save the new arrangement and calculate the resulting profit. Otherwise, if possible, implement the following sub-moves regardless of an increase or decrease in profit. For each of these sub-moves, check if each adjustment described below can be made. If so, make the adjustment, and check if there is enough space on shelf $j_{1} k_{1}$ and shelf $j_{2} k_{2}$ to swap $i_{1}$ and $i_{3}$. If there is enough space, make the swap, save the new arrangement and calculate the resulting profit.

If $x_{i_{1} j_{1} k_{1}}>L_{i_{1}}$ then $x_{i_{1} j_{1} k_{1}}=x_{i_{1} j_{1} k_{1}}-1$

If $x_{i_{3} j_{2} k_{2}}>L_{i_{3}}$ then $x_{i_{3} j_{2} k_{2}}=x_{i_{3} j_{2} k_{2}}-1$

If $x_{i_{1} j_{1} k_{1}}>L_{i_{1}}$ and $x_{i_{3} j_{2} k_{2}}<U_{i_{3}}$ then $x_{i_{1} j_{1} k_{1}}=x_{i_{1} j_{1} k_{1}}-1$ and $x_{i_{3} j_{2} k_{2}}=X_{i_{3} j_{2} k_{2}}+1$

If $x_{i_{1} j_{1} k_{1}}<U_{i_{1}}$ and $x_{i_{3} j_{2} k_{2}}>L_{i_{3}}$ then $x_{i_{1} j_{1} k_{1}}=x_{i_{1} j_{1} k_{1}}+1$ and $x_{i_{3} j_{2} k_{2}}=x_{i_{3} j_{2} k_{2}}-1$

If $x_{i_{1} j_{1} k_{1}}<U_{i_{1}}$ and $x_{i_{3} j_{2} k_{2}}<U_{i_{3}}$ then $x_{i_{1} j_{1} k_{1}}=x_{i_{1} j_{1} k_{1}}+1$ and $X_{i_{3} j_{2} k_{2}}=x_{i_{3} j_{2} k_{2}}+1$

If $x_{i_{1} j_{1} k_{1}}>L_{i_{1}}$ and $x_{i_{3} j_{2} k_{2}}>L_{i_{3}}$ then $x_{i_{1} j_{1} k_{1}}=x_{i_{1} j_{1} k_{1}}-1$ and $x_{i_{3} j_{2} k_{2}}=x_{i_{3} j_{2} k_{2}}-1$

\section{Interchange $i_{2}$ (on shelf $j_{1} k_{1}$ ) and $i_{4}$ (on shelf $j_{2} k_{2}$ )}

If $x_{i_{2} a}=L_{i_{2}}$ and $x_{i_{4} b}=L_{i_{4}}$, and there is enough space on each shelf part to swap $i_{2}$ and $i_{4}$, make the swap, save the new arrangement and calculate the resulting profit. Otherwise, if possible, implement the following sub-moves only if it results in an increase in profit. For each of these sub-moves, check if each adjustment described below can be made. If so, make the adjustment, and check if there is enough space on shelf $j_{1} k_{1}$ and shelf $j_{2} k_{2}$ to swap $i_{2}$ and $i_{4}$. If there is enough space, make the swap, save the new arrangement and calculate the resulting profit.

If $x_{i_{2} j_{1} k_{1}}>L_{i_{2}}$ then $x_{i_{2} j_{1} k_{1}}=x_{i_{2} j_{1} k_{1}}-1$

If $x_{i_{4} j_{2} k_{2}}>L_{i_{4}}$ then $x_{i_{4} j_{2} k_{2}}=x_{i_{4} j_{2} k_{2}}-1$

If $x_{i_{2} j_{1} k_{1}}>L_{i_{1}}$ and $x_{i_{4} j_{2} k_{2}}<U_{i_{4}}$ then $x_{i_{2} j_{1} k_{1}}=x_{i_{2} j_{1} k_{1}}-1$ and $x_{i_{4} j_{2} k_{2}}=x_{i_{4} j_{2} k_{2}}+1$

If $x_{i_{2} j_{1} k_{1}}<U_{i_{2}}$ and $x_{i_{4} j_{2} k_{2}}>L_{i_{4}}$ then $x_{i_{2} j_{1} k_{1}}=x_{i_{2} j_{1} k_{1}}+1$ and $x_{i_{4} j_{2} k_{2}}=x_{i_{4} j_{2} k_{2}}-1$

If $x_{i_{2} j_{1} k_{1}}<U_{i_{2}}$ and $x_{i_{4} j_{2} k_{2}}<U_{i_{4}}$ then $x_{i_{2} j_{1} k_{1}}=x_{i_{2} j_{1} k_{1}}+1$ and $x_{i_{4} j_{2} k_{2}}=x_{i_{4} j_{2} k_{2}}+1$

\section{RemoveLeastProfitable}

We propose this move which is intended to prevent the algorithm getting stuck in a local optimum. For one out of ten randomly chosen shelf parts, find the product $i_{1}$ which has more than the minimum required facings $x_{i j k}>L_{i}$ and also contributes with the least profit $\varphi_{i j k}$ and reduce the number of its facings by one $x_{i j k}=x_{i j k}-1$. The aim is to disturb the solution so that the other moves can ultimately lead to a much better overall profit. Reducing the number of facings for the least profitable products leaves more space to allocate additional facings of higher profitable products and thus increase the overall profit.

\section{EXPERIMENTS AND RESULTS}

\subsection{Experimental Setting}

The experiments were carried out using data kindly provided by the management of the UN shop. Two problem instances were made available to us, a small one with 135 products and 15 shelf parts and a large one with 907 products and 114 shelf parts. The characteristics of each problem instance are outlined below.

Small Problem. Includes 135 products and 15 shelf parts ( 5 shelves). The total shelf space available is 33 meters. The minimum number of facings required for each product is 1 . The maximum number of facings required for each product ranges from 2 to 11 . The average width of a product facing is 0.11 meters.

Large problem. Includes 907 products and 114 shelf parts (38 shelves). The total shelf space available is 248 meters. The minimum number of facings required for each product is 1 . The maximum number of facings required for each product ranges from 2 to 13 . The average width of a product facing is 0.13 meters.

\subsection{Allocation Phase}

Only the Arrangement Random Choice heuristic produces different arrangements in each run. Therefore, this heuristic was executed 20 times and the average profit was computed. The profits achieved by each of the initialisation heuristics on the small and large problems are shown in Table 1 together with the profit for the current arrangement. The results for the large problem are expected. It is clear that an arrangement sorted by profit offers the highest overall profit. The arrangement sorted by size produces just a slightly higher profit than the random and current arrangements. The current and random arrangements produce very similar profits. This suggests that the UN shop management has not taken much care in creating their arrangement. It seems that products are placed in a random fashion rather than being organised by some logical heuristic. We would expect similar results on the small problem. However, it appears that on a small scale, the supermarket has allocated their products fairly efficiently. Note that the three initialisation heuristics produce an arrangement very quickly even for the large problem. 


\begin{tabular}{c|c|c|c|c} 
& Current & Profit & Size & Random \\
\hline Small Problem & $78.646(\mathrm{~N} / \mathrm{A})$ & $77.119(0.07)$ & $71.360(0.06)$ & $71.806(0.07)$ \\
Large Problem & $2463.053(\mathrm{~N} / \mathrm{A})$ & $2959.334(3.55)$ & $2574.381(3.33)$ & $2513.546(4.07)$
\end{tabular}

Table 1: Profit of the arrangements generated by the initialisation heuristics and profit of the current arrangement. The computation time (in seconds) for the three initialisation heuristics is shown in brackets.

\subsection{Adjustment Phase}

Each adjustment heuristic was executed alone for 30 seconds, this was repeated 20 times for each of the four initial arrangements generated above. The aim of this experiment was to assess the effectiveness of each adjustment heuristic on improving the overall profit of the initial solution. The summarised results are shown in Table 2. Using these results, we assigned priorities to each adjustment heuristic in the local search when selecting the next move to explore. We assigned probability equal to 0.35 to each of the two best moves (MultiShift and AdjustInter) and probability of 0.15 to each of the two other moves (AdjustIn and Swap).

Then, the overall Adjustment Phase incorporating the five adjustment heuristics was executed 15 times for each of the initial arrangements and each of the two problems. Each run took 1000 seconds for the small problem and 8000 seconds for the large problem. This execution time was set based in our preliminary experiments in which we observed that usually after around 600 seconds for the small problem and 7000 seconds for the large problem no further improvement was achieved. In each iteration of this Adjustment Phase, one of the Swap, AdjustIn, MultiShift or AdjustInter heuristics is selected with the probability as mentioned above. The RemoveLeastProfitable move was applied whenever after one tenth of the overall execution time (100 seconds for the small problem and 800 seconds for the large problem) no improvement in the current arrangement was observed.

The results of these experiments are shown in Table 3 for both problems. We can see that for the small problem, the profit increase achieved by the Adjustment Phase is fairly similar regardless of the initialisation heuristic used and also quite large (around 50\% improvement over the initial profit) in all cases. This gives an indication that the adjustment phase is capable of making substantial improvements over the initial arrangements including the manual current arrangement. For the large problem, we can see that profits have almost doubled from the initial arrangements. It should be noted that since adjustment heuristic moves are not restricted to product category, the resulting arrangement contains products from different categories interspersed. While it is important for the customer to be able to find products according to categories, placing product category restrictions in a small shop can greatly affect the arrangement possibilities available. A small shop has very limited shelf space available, fewer number of products and therefore, there are only a few products within each category. We argue, with some support from the UN shop manager, that in a small shop, product category restriction is not crucial since most products are likely to be visible. On the other hand, we should remember that customer satisfaction plays a big role in profits, so the arrangement must be also such that it is convenient for the customer. Further assessment of the proposed arrangements by the shop manager is planned.

\subsection{Generated Planograms}

Once the final arrangement of products on shelves is created and evaluated, the termination phase also generates the graphical visualisation of the proposed solution in the form of a simple planogram. This is the tool that managers of retail shops consider important to aid them in deciding the best way to utilise the available shelf space. An example of the planograms generated with our system is shown in Figure 1.

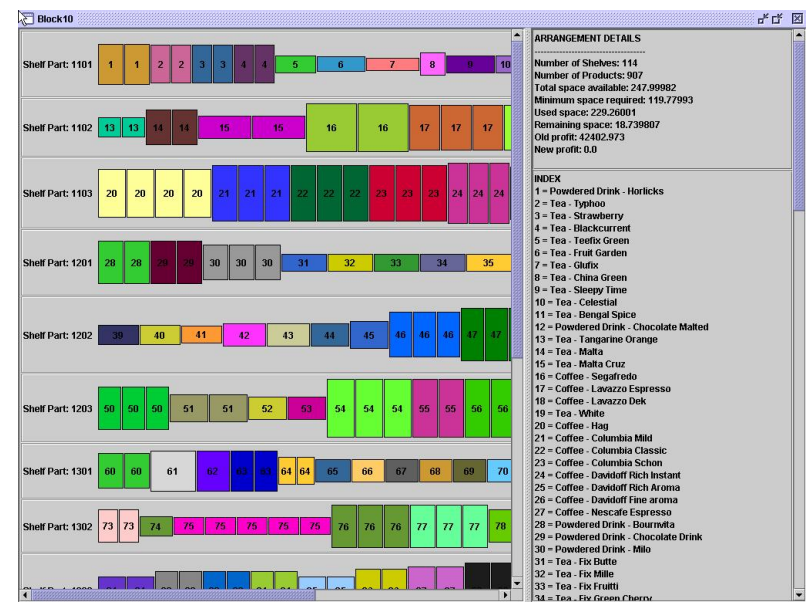

Figure 1: Example of planogram generated with the proposed system for the large problem.

\section{CONCLUSIONS}

The optimisation of shelf space allocation is a difficult problem and a very important area of research. Recent work has concentrated on the case of large retail shops like big supermarkets. The aim of this paper was to produce an efficient optimisation model for the case of small retail shops. Real data taken from a small retailer and communications with the shop manager helped us to gather the relevant information to approach this problem. We have designed a heuristic method inspired by the work of previous researchers but adapted to the specific needs of small retail shops. The proposed heuristic creates an initial arrangement and then uses adjustment moves in order to iteratively improve the profit of the current solution. We tested the method on two problem instances, one small problem involving 135 products on 15 shelves and one large problem involving 907 products on 114 shelves. Our results show that the method is capable of generating much improved arrangements in terms of the overall profit. We also created a tool to visualise the proposed arrangements in the form of simple planograms.

This initial investigation illustrates that a simple and lowcost shelf space allocation decision support system focused 


\begin{tabular}{l|c|c|c|c|c|c|c|c|} 
& \multicolumn{5}{|c|}{ Small Problem } & \multicolumn{5}{c|}{ Large Problem } \\
& Current & Profit & Size & Random & Current & Profit & Size & Random \\
\hline Swap & 7.168 & 5.167 & 5.874 & 6.535 & 83.651 & 57.086 & 83.806 & 72.477 \\
\hline AdjustIn & 11.768 & 23.086 & 25.164 & 23.472 & 133.850 & 434.227 & 432.833 & 396.897 \\
\hline MultiShift & 17.574 & 28.250 & 30.734 & 29.227 & 202.434 & 394.433 & 396.424 & 442.737 \\
\hline AdjustInter & 20.986 & 20.621 & 27.262 & 27.446 & 243.619 & 94.790 & 221.183 & 105.923
\end{tabular}

Table 2: Average increase in profit achieved by the adjustment heuristics on each problem.

\begin{tabular}{l|c|c|c|c|c|c|c|c|} 
& \multicolumn{4}{|c|}{ Small Problem } & \multicolumn{4}{c|}{ Large Problem } \\
& \multicolumn{2}{|c|}{ Profit Increase } & \multicolumn{2}{c|}{ Overall Profit } & \multicolumn{3}{c|}{ Profit Increase } & \multicolumn{2}{c|}{ Overall Profit } \\
& Mean & Std Dev & Initial & Final & Mean & Std Dev & Initial & Final \\
\hline Current & 29.064 & 0.7789 & 78.646 & 107.710 & 2386.334 & 51.198 & 2463.053 & 4849.387 \\
Profit & 30.93 & 0.9372 & 77.119 & 108.049 & 1869.772 & 27.562 & 2959.334 & 4829.106 \\
Size & 37.194 & 0.8388 & 71.360 & 108.554 & 2391.472 & 23.961 & 2574.381 & 4965.853 \\
Random & 36.253 & 0.5154 & 71.806 & 108.059 & 2191.735 & 36.024 & 2513.546 & 4705.281
\end{tabular}

Table 3: Summary of results achieved by the heuristic method on the small and large problems.

on small retailers will not only be appealing but also encourage shop managers to use these tools to their advantage. There are a number of issues that demand further investigation. For example, modify the RemoveLeastProfitable move to overcome the local optimum more effectively. Currently, this move removes one product from within ten shelves. Even for the large problem with 114 shelves, this means that only 11 (out of the 907) products are removed. Perhaps removing more products could result in more space being available and better adjustments made later. Another issue is the objective function which at present, is based on the perceived profitability as measured by the shop manager. Perhaps, further evaluation on the proposed arrangements in the real world could also suggest other criteria that should be considered when evaluating the quality of the generated arrangements. Also, much further experimentation should be carried out on more instances and more scenarios. We intend to continue with this research and collect or generate data that reflects the real world problem faced by small retail shops.

\section{ACKNOWLEDGMENTS}

We thank the management of the UN shop in Vienna for kindly providing the data and information for the work presented in this paper. We are also grateful to the anonymous referees for their comments and feedback.

\section{REFERENCES}

[1] E. Anderson and H. Amato. A mathematical model for simultaneously determining the optimal brand collection and display area allocation. Operations Research, 22(1):13-21, 1974.

[2] N. Borin, P. Farris, and J. Freeland. A model for determining retail product category assortment and shelf space allocation. Decision sciences, 25(3):359-384, 1994.

[3] F. Buttle. Retail space allocation. International Journal of Physics Distribution and Material Management, 14(4):3-23, 1984.

[4] J. Cairns. Allocate space for maximum profits. Journal of Retailing, 39(2):41-55, 1963.

[5] M. Corstjens and P. Doyle. A model for optimizing retail space allocations. Management Science, 27(7):822-833, 1981.

[6] X. Dreze, S. Hoch, and M. Purk. Shelf management and space elasticity. Journal of Retailing, 70(4):301-326, 1994.

[7] P. Hansen and H. Heinsbroek. Product selection and space allocation in supermarkets. European journal of operational research, 3(6):474-484, 1979.

[8] A. Lim, B. Rodrigues, and X. Zhang. Metaheuristics with local search techniques for retail shelf-space optimization. Management science, 50(1):117-131, 2005.

[9] M. Yang. An efficient algorithm to allocate shelf space. European journal of operational research, 131:107-118, 2001.

[10] M. Yang and W. Chen. A study on shelf space allocation and management. International journal of production economics, 60-61:309-317, 1999.

[11] F. Zufryden. A dynamic programming approach for production selection and supermarket shelf-space allocation. Journal of the operational research society, 37(4):413-422, 1986. 